\title{
Gastric cancer invading the pancreas
}

\section{To the Editor:}

I read with interest the recent article by Saka et al. [1]. The authors reviewed 195 patients with gastric cancer macroscopically invading the pancreatic head (T4) and examined 23 patients who underwent pancreaticoduodenectomy (PD) with curative intent.

They showed that when there was no paraaortic lymph node metastasis, peritoneal dissemination, or positive lavage cytology, the 5-year-survival rate and median survival time were $47.4 \%$ and 26 months, respectively $(n=15)$. They recommended that PD should be performed for patients with tumor invading the pancreatic head.

However, their data showed that in five patients who survived more than 3 years there was no histologic evidence of pancreatic invasion (Table 1), except in one patient with cancer of the gastric remnant (case 21). When 16 patients who had pT4 tumor were reviewed, it was found that 13 died of recurrence with median survival time of 13 months, two were alive with recurrence, and only one did not have recurrence (case 6).

These results show that when gastric cancer grossly invaded the pancreas (T4), candidates for curative resection were few $(12 \%)$, and when the tumor invaded the pancreatic tissue (pT4), recurrence was frequent (94\%), even after radical operations. Considering the high operative morbidity (74\%), the authors should not recommend $\mathrm{PD}$.

Sugawara et al. [2] examined 114 patients with gastric cancer grossly invading the pancreas or duodenum and compared those with PD and those without PD. Although the 5-year-survival rate of patients with T4 tumor was different ( $37 \%$ vs $13 \%)$, that with pT4 tumor was not different $(12 \%$ vs $0 \%)$.

They concluded that PD was indicated for patients with gastric cancer grossly invading the pancreatic head (T4); however, the prognosis was poor and intensive chemotherapy was necessary when pancreatic invasion was histologically positive (pT4).
Because the candidates for PD were few $(23 / 9349=$ $0.2 \%)$ and operative morbidity was high (74\%) even in a specialized center of Japan [1], aggressive surgical procedures for advanced gastric cancer would be better performed by skilled surgeons or in high-volume hospitals [3-5].

Yosuke Adachi

Oncologic Surgery, Gifu University Yanagido 1-1, Gifu 501-1194, Japan

\section{References}

1. Saka M, Mudan SS, Katai H, Sano T, Sasako M, Maruyama K. Pancreaticoduodenectomy for advanced gastric cancer. Gastric Cancer 2005;8:1-5.

2. Sugawara G, Yamaguchi A, Isogai M, Harada T, Kaneoka Y, Suzuki M, et al. A clinical study on 36 cases of pancreaticoduodenectomy for gastric cancer (in Japanese). J Jpn Surg Assoc 2002;63:2883-9.

3. Adachi Y, Mimori K, Mori M, Maehara Y, Sugimachi K. Morbidity after D2 and D3 gastrectomy for node-positive gastric carcinoma. J Am Coll Surg 1997;184:240-4.

4. Hannan EL, Radzyner M, Rubin D, Dougherty J, Brennan MF. The influence of hospital volume and surgeon volume on inhospital mortality for colectomy, gastrectomy, and lung lobectomy in patients with cancer. Surgery 2002;131:6-15.

5. Birkmeyer JD, Siewers AE, Finlayson EVA, Stukel TA, Licas FL, Batista I, et al. Hospital volume and surgical mortality in the United States. New Engl J Med 2002;346:112837.

\section{Authors' reply}

To the Editor:

We appreciate Dr. Adachi's response to our article. He says that we should not recommend PD because of the very poor prognosis of pT4 patients and high operative mortality. His criticism is based on the pathological findings after operation. The prognosis of pT4 tumors 
is indeed poor. However, surgeons have to decide the operative method during operation and not after operation. Because pre- and intra-operative diagnosis of pancreatic invasion is inaccurate, we cannot exclude pT4 patients during operation. As we mentioned in our article, there was no significant difference in survival after $\mathrm{R} 0$ resection between patients who required $\mathrm{PD}$ and those who did not, and the survival after R0 resection was fair. We should emphasize again that to achieve $\mathrm{R} 0$ resection is an important objective irrespective of whether PD is performed. However, surgeons have to remember that, owing to desmoplastic reaction sur- rounding the tumor, they cannot avoid PD for R0 resection in some cases, even though there is no pathological pancreatic invasion. Therefore, we believe that PD is still worthwhile, and should be performed in institutions where PD for pancreatic cancer is frequently performed. Patients with pT4 disease would be good candidates for adjuvant chemotherapy, as Dr. Adachi comments.

Hitoshi Katai

National Cancer Center Hospital 5-1-1 Tsukiji, Chuo-ku, Tokyo 104-0045, Japan 